\title{
Application of Constrained Linear MPC to a Spray Dryer
}

Petersen, Lars Norbert; Poulsen, Niels Kjølstad; Niemann, Hans Henrik; Utzen, Christer; Jørgensen, John Bagterp

\section{Published in:}

Proceedings of the IEEE Conference on Control Applications (CCA), 2014

Link to article, DOI:

10.1109/CCA.2014.6981616

Publication date:

2014

Link back to DTU Orbit

Citation (APA):

Petersen, L. N., Poulsen, N. K., Niemann, H. H., Utzen, C., \& Jørgensen, J. B. (2014). Application of Constrained Linear MPC to a Spray Dryer. In Proceedings of the IEEE Conference on Control Applications (CCA), 2014 (pp. 2120-2126). IEEE. https://doi.org/10.1109/CCA.2014.6981616

\section{General rights}

Copyright and moral rights for the publications made accessible in the public portal are retained by the authors and/or other copyright owners and it is a condition of accessing publications that users recognise and abide by the legal requirements associated with these rights.

- Users may download and print one copy of any publication from the public portal for the purpose of private study or research.

- You may not further distribute the material or use it for any profit-making activity or commercial gain

- You may freely distribute the URL identifying the publication in the public portal 


\title{
Application of Constrained Linear MPC to a Spray Dryer
}

\author{
Lars Norbert Petersen ${ }^{1,2}$, Niels Kjølstad Poulsen ${ }^{1}$, Hans Henrik Niemann ${ }^{3}$, \\ Christer Utzen $^{2}$ and John Bagterp Jørgensen ${ }^{1}$
}

\begin{abstract}
In this paper we develop a linear model predictive control (MPC) algorithm for control of a two stage spray dryer. The states are estimated by a stationary Kalman filter. A non-linear first-principle engineering model is developed to simulate the spray drying process. The model is validated against experimental data and able to precisely predict the temperatures, the air humidity and the residual moisture in the dryer. The MPC controls these variables to the target and reject disturbances. Spray drying is a cost-effective method to evaporate water from liquid foods and produces a free flowing powder. The main challenge of spray drying is to meet the residual moisture specification and prevent powder from sticking to the chamber walls. By simulation we compare the performance of the MPC against the conventional PID control strategy. During an industrially recorded disturbance scenario, the MPC increases the production rate by $7.9 \%$, profit of production by $8.2 \%$ and the energy efficiency by $4.1 \%$ on average.
\end{abstract}

\section{INTRODUCTION}

In 2015 the milk quota system in the European Union will be completely liberalized. The expected effect of this liberalization is that the milk production increases significantly. In some countries up to $50 \%$ in 2020 [1]. The milk production expansion will mainly affect the milk powder production due to the short shelf life of dairy products. Consequently, the capacity for production of milk powder will need to increase [1]. Industry studies show that advanced control is capable of increasing the capacity of spray dryers by up to $20 \%$ [2]. Optimal control is therefore an effective way to leverage the future production increase.

The main objective in controlling a spray dryer is to minimize the energy consumption while bringing the residual moisture in the powder below the specification and avoid that the powder sticks to the chamber walls. The dryers are generally very large. These may have a feed capacity of up to 4.4 million 1/day of raw milk and require $7 \mathrm{MW}$ of power. Therefore, even small improvement in operation will have a large impact on the profitability. The challenge is in maintaining optimal operation since to do so the dryer must be constantly adjusted to variations in the feed concentration and ambient air humidity.

Conventional PID control of spray dryers keeps inletand outlet temperatures constant during operation. This is a simple approach, but known to be insufficient at controlling

1 Department of Applied Mathematics and Computer Science, Technical University of Denmark, Kgs. Lyngby, Denmark $\{$ Inpe, nkpo, jbjo\} adtu.dk

2 GEA Process Engineering A/S, Søborg, Denmark christer.utzen@gea. com

3 Department of Electrical Engineering, Technical University of Denmark, Kgs. Lyngby, Denmark hhn@elektro.dtu.dk

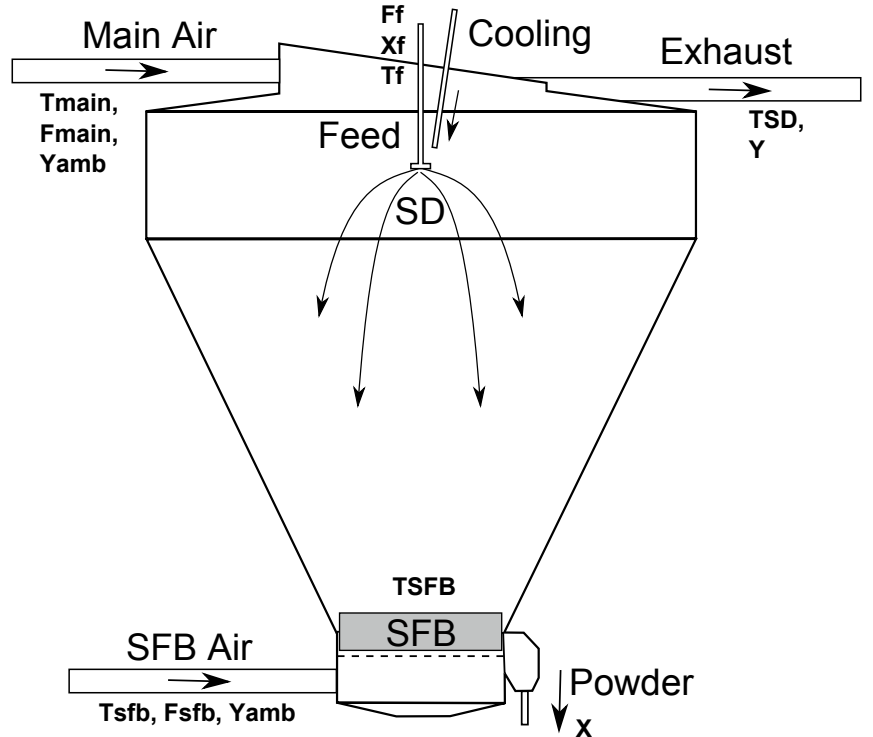

Fig. 1: Illustration of the spray chamber (SD) and the static fluid bed (SFB) stages of a two stage spray dryer. Hot air is let into the upper section of the drying chamber where the nozzles disperse the liquid feed. The droplets dry into powder particles in these two stages.

the residual moisture and prevent powder from sticking to the chamber walls [3]. The variations in residual moisture of the produced powder reflects that the conventional PID controller cannot reject disturbances such as feed concentration variations and air humidity variations. Still, this conventional control strategy is the de facto standard in the industry. The MPC proposed in this paper constantly adjusts the manipulated variables such that the residual moisture, the exhaust air temperature and exhaust air humidity is controlled to a reference. The temperature and humidity in the dryer are closely correlated to stickiness of powder. Therefore, controlling the temperature and humidity indirectly controls the stickiness and deposits of the powder in the dryer. The residual moisture must be controlled to specifications to avoid bacterial growth and preserve the milk powder. Conventional control must frequently be manually adjusted in order to avoid stickiness of the powder and produce at specified residual moisture content.

\section{A. Process Description}

The two stage spray dryer is an energy efficient dryer and is often used for production of milk and other food powders. Fig. 1 illustrates the spray chamber (SD) and static fluid 
bed stage (SFB). The main hot air is let into the upper section of the drying chamber (SD) around the high pressure nozzles. The nozzles disperse the liquid feed into droplets. The heat is transferred from the hot air to the droplets, and due to this transfer water evaporates from the droplets. In that process, the air temperature and the residual moisture of the droplets decrease. The dried product then enters the static fluid bed (SFB) where it is dried further. After drying in the $\mathrm{SFB}$, the powder is transported to the external vibrating fluid bed (VFB) for gentle drying and cooled to the temperature desired for handling and storage. The VFB is not illustrated in Fig. 1.

\section{B. Modelling and Control}

Modelling of the drying process in spray dryers varies in complexity and purpose. First order transfer function models as well as first principles engineering models for control purposes are proposed [4], [5]. [6] proposes a dynamic model for a complete multi-stage dryer. A number of papers present control strategies for spray dryers. The focus varies from pressure control of the chamber pressure to MPC control of the residual moisture based on industrial software [2]. [7] provides a detailed review on the status and future of advanced control for spray dryers. In [5] a feed forward approach is made to help control the residual moisture. Setpoint tracking MPC is a standard methodology for optimizing the operation of processes and a recognised control method in both academia and industry due to its ability to handle coupled dynamics, time delays, constraints and feed forward of measured disturbances [8], [9]. In this methodology the economics of operation is optimized in a separate realtime steady-state optimization (RTO) layer [10]. Recent advances has shifted to combine these two layers into a single economically optimizing (E-)MPC layer. [11] use nonlinear (E-)MPC for economic optimization of a two stage spray dryer.

\section{Content}

In this paper we investigate the potential of optimizing the spray drying process by linear MPC. We compare the linear MPC to the conventional PID based control method. The MPC is composed of an optimal regulator and a state estimator. The regulator has the structure described in [12][14], with the modification that we use a deterministic linearised first principles engineering model instead of an innovation form state space model identified from data. The model used for simulation is also based on the non-linear first principles engineering model. The proposed model is identified and validated against two independent experimental datasets provided by GEA Process Engineering A/S.

\section{Organization}

The paper is organized as follows. In Section II we describe the model and show its accuracy by comparing its output to experimental data. Section III presents the regulator and state estimator. In Section IV we present two simulations to validate the MPC and show the benefit of optimizing the two stage spray dryer. Conclusions are given in Section V.

\section{SPRAY DRYER MODEL}

In this section, we present the first principles engineering model for a Multi-Stage Dryer type $20\left(\mathrm{MSD}^{\mathrm{TM}}-20\right)$, i.e. a medium-sized two stage spray dryer, made available by GEA Process Engineering A/S. The dryer has a maximum water evaporation capacity of approximately $125 \mathrm{~kg} / \mathrm{h}$. The data for identification is based on drying of sugar water, maltodextrin DE-18. We use maltodextrin, because milk is difficult to handle over longer periods due to natural deterioration and maltodextrin DE-18 has the same drying properties as skim milk [15].

\section{A. Mass- and Energy Balances}

The model consists of four states. The states are the spray dryer temperature, $T_{S D}$, the static fluid bed temperature, $T_{S F B}$, the humidity in the air of the spray dryer, $Y$, and the moisture in the powder, $X$. The lumped energy and mass balances describing the evolution of the states are

$$
\begin{aligned}
& C_{a} \frac{d T_{S D}}{d t}=-\lambda R_{w}+H_{a_{i n}}-H_{a_{o u t}}-Q_{e x c}-Q_{l a} \\
& C_{b} \frac{d T_{S F B}}{d t}=H_{b_{i n}}-H_{b_{o u t}}+Q_{e x c}-Q_{l b} \\
& m_{d a} \frac{d Y}{d t}=\left(F_{\text {main }}+F_{s f b}\right)\left(Y_{a m b}-Y\right) \\
& \quad+F_{a d d}\left(Y_{a d d}-Y\right)+R_{w} \\
& m_{s} \frac{d X}{d t}=F_{s}\left(X_{f}-X\right)-R_{w}
\end{aligned}
$$

where

$$
\begin{aligned}
H_{a_{\text {in }}}= & F_{\text {main }}\left(c_{d a}+c_{v} Y_{a m b}\right)\left(T_{\text {main }}-T_{0}\right)+ \\
& F_{a d d}\left(c_{d a}+c_{v} Y_{a d d}\right)\left(T_{a d d}-T_{0}\right) \\
H_{a_{\text {out }}}= & \left(F_{\text {main }}+F_{s f b}+F_{a d d}\right) \\
& \left(c_{d a}+c_{v} Y\right)\left(T_{S D}-T_{0}\right) \\
H_{b_{\text {in }}}= & F_{s f b}\left(c_{d a}+c_{v} Y_{a m b}\right)\left(T_{s f b}-T_{0}\right) \\
H_{b_{\text {out }}}= & F_{s f b}\left(c_{d a}+c_{v} Y_{a m b}\right)\left(T_{S F B}-T_{0}\right) \\
Q_{e x c}= & k_{1}\left(T_{S D}-T_{S F B}\right)+k_{2} X_{f}+k_{3} T_{f}-k_{4} \\
Q_{l a}= & k_{5}\left(T_{S D}-T_{a m b}\right) \\
Q_{l b}= & k_{6}\left(T_{S F B}-T_{a m b}\right) \\
F_{s}= & F_{f} X_{f} /\left(X_{f}+1\right)
\end{aligned}
$$

The air and the product temperatures are assumed in equilibrium in both stages, i.e. $T_{S D}$ and $T_{S F B}$ are each identical to the powder temperatures in the SD- and the SFB-stage, respectively. $H_{a_{\text {in }}}, H_{a_{\text {out }}}, H_{b_{\text {in }}}$ and $H_{b_{\text {out }}}$ are the enthalpies of humid air in and out of the SD and the SFB stage, respectively. The reference temperature is $T_{0}=25^{\circ} \mathrm{C} . C_{a}$ and $C_{b}$ is the heat capacity of the hold-up of air and powder. $F_{\text {main }}$ and $F_{s f b}$ are dry basis inlet air flows. The parameters $Y_{a d d}$ and $F_{a d d}$ are used to correct for air leakage and other un-modeled inlet air flows such as nozzle cooling air. $Q_{\text {exc }}$ is the heat exchange between the SD and the SFB stages. $Q_{l a}$ and $Q_{l b}$ are heat losses to the surroundings. $\lambda R_{w}$ is the heat of evaporation. We assume that the evaporation only takes place in the SD stage. $X_{f}$ is the dry base feed concentration and $T_{f}$ is the feed temperature. $m_{s}$ is the dry 
mass of powder and $m_{d a}$ is the mass of dry air. The latent heat of vaporization, $\lambda$, and the heat capacities, $c_{d a}$ and $c_{v}$, are calculated according to [16].

\section{B. Drying Rate}

The drying rate of the product, $R_{w}$, is an important parameter of the model. The thin layer equation describes the product drying rate, $R_{w}$, well [17]

$$
R_{w}=D_{\text {diff }}\left(X-X_{e q}\right) m_{s}
$$

$D_{\text {diff }}$ is an experimentally determined constant called the drying constant or diffusivity [18]. It may depend on both the temperature and the moisture of the powder, but in the present study it is assumed constant.

The equilibrium moisture, $X_{e q}$, describes the moisture content at which water cannot be evaporated from the powder any longer. The Guggenheim-Anderson-de Boer (GAB) relation is used to describe the equilibrium moisture content [19] and is fitted to laboratory data obtained specifically from maltodextrin to get

$$
X_{e q}=\frac{C \cdot K \cdot X_{m} \cdot R H}{(1-K \cdot R H)(1-K \cdot R H+C \cdot K \cdot R H)}
$$

where $X_{m}=0.030723, C=2.6535 \cdot 10^{-7} \exp \left(\frac{6292.1}{T}\right)$ and $K=0.057882 \exp \left(\frac{945.16}{T}\right) \cdot X_{m}, C$ and $K$ are GAB constants related to monolayer and multilayer properties. In the computation of $X_{e q}, T=T_{S F B}$ and $R H=$ $Y /(18.02 / 28.97+Y) \cdot p_{c} / p_{\text {vapsat }}(T) \cdot p_{c}$ is the chamber pressure and $p_{\text {vapsat }}$ is the saturated vapor pressure.

\section{Performance}

The energy efficiency, the profit of operation and the product flow rate are the three key performance indicators (KPIs) for evaluation of the performance of a spray dryer. There are a number of energy efficiency indices to measure the energy performance of a dryer. We adopt the definition given by [20]

$$
\eta=\frac{\lambda F_{s}\left(X_{f}-X\right)}{\Delta H}
$$

Here $\lambda F_{s}\left(X_{f}-X\right)$ is the energy used to evaporate water and $\Delta H$ is the total energy supplied to the dryer given by

$$
\Delta H=F_{\text {main }}\left(h_{a_{i n}}-h_{a m b}\right)+F_{s f b}\left(h_{b_{i n}}-h_{a m b}\right)
$$

in which $h_{a m b}=\left(c_{d a}+c_{v} Y_{a m b}\right)\left(T_{a m b}-T_{0}\right)$. The profit from operating the spray dryer is the value of the product minus the raw material and energy costs.

$$
P=p_{p} F_{s}(1+X)-p_{f} F_{s}\left(1+X_{f}\right)-p_{H} \Delta H
$$

The price of the produced powder is $p_{p}=4.47 \$ / \mathrm{kg}$, the price of feed material is $p_{f}=0.447 \$ / \mathrm{kg}$, and the price of energy is $p_{H}=3.4873 \cdot 10^{-5} \$ / \mathrm{KJ}$. The prices are selected to reflect the industrial prices of natural gas and the price of the powder. The flow rate of powder is $F_{p}=F_{s}(1+X)$.
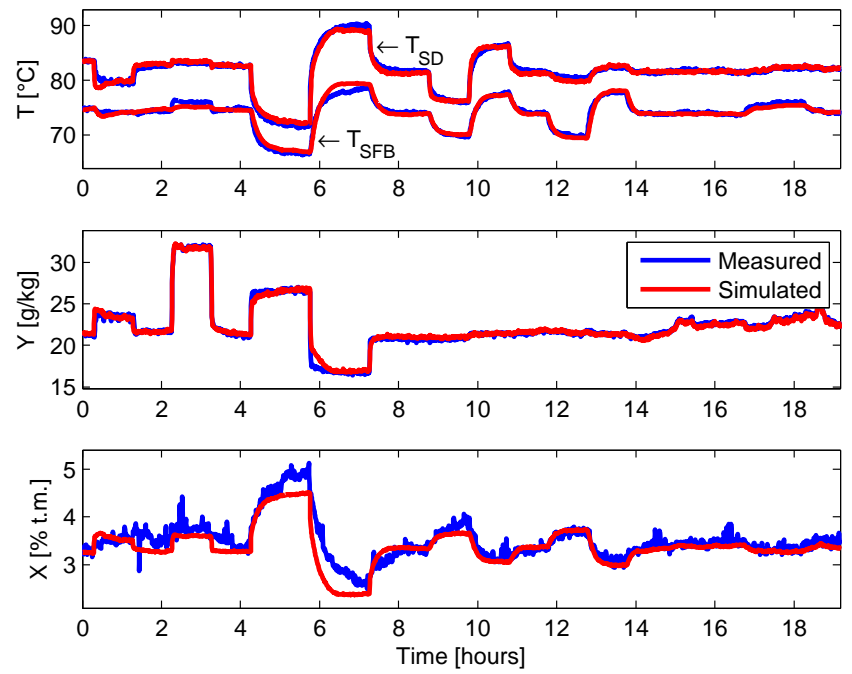

Fig. 2: Model validation dataset. The disturbances i.e. feed concentration and ambient air humidity are altered from $t=$ 0 to $t=4$. The feed flow is altered between $t=4$ and $t=8$. The main inlet air temperature, the SFB inlet air temperature and SFB air flow rate is changed between $t=8$ to $t=15$.

\section{Parameter Estimation}

The parameters, $\theta$, in the model (1)-(4) are estimated from data (not shown here) by minimizing the least squares simulation error (not 1-step prediction error). The model is validated against the data in Fig. 2. Generally the model fits the data well. Table I provides the parameters and all parameters are significantly identified. The mass of dry powder and dry air are determined from physical considerations and fixed to $m_{d a}=10 \mathrm{~kg}$ and $m_{s}=15 \mathrm{~kg}$.

The model of the dryer is a deterministic system of ordinary differential equations, i.e.

$$
\frac{d \bar{x}(t)}{d t}=f(\bar{x}(t), u(t), d(t), \theta)
$$

in which

$$
\begin{aligned}
& \bar{x}=\left[\begin{array}{llll}
T_{S D} & T_{S F B} & Y & X
\end{array}\right]^{T} \\
& u=\left[\begin{array}{lll}
F_{f} & T_{\text {main }} & T_{s f b}
\end{array}\right]^{T} \\
& d=\left[\begin{array}{llllll}
F_{\text {main }} & F_{s f b} & X_{f} & T_{f} & T_{a m b} & Y_{a m b}
\end{array}\right]^{T}
\end{aligned}
$$

$\bar{x}$ is the state vector, $u$ is the manipulated input vector, $d$ is the disturbance vector and $\theta$ is the parameter vector. The measurement vector, $y$, and the controlled output vector, $z$, are

$$
y=\left[\begin{array}{llll}
T_{S D} & T_{S F B} & Y & X
\end{array}\right]^{T}, \quad z=\left[\begin{array}{lll}
T_{S D} & Y & X
\end{array}\right]^{T}
$$

\section{E. Stochastic Model}

The deterministic system in (8) is augmented by two stochastic terms. Consequently, the state evolution and measurement equation of the dryer is described by

$$
\bar{x}\left(t_{k}+T_{s}\right)=F\left(\bar{x}\left(t_{k}\right), u\left(t_{k}\right), d\left(t_{k}\right), \theta\right)+\bar{w}\left(t_{k}\right)
$$


TABLE I: Estimated parameters for the model (1)-(4)

\begin{tabular}{l|l|l||l|l|l}
\hline Symbol & Value & Unit & Symbol & Value & Unit \\
\hline \hline$C_{a}$ & 68.266 & $\mathrm{KJ} / \mathrm{K}$ & $F_{a d d}$ & $256.46 / 3600$ & $\mathrm{~kg} / \mathrm{s}$ \\
\hline$C_{b}$ & 141.36 & $\mathrm{KJ} / \mathrm{K}$ & $Y_{a d d}$ & $8.1799 \cdot 10^{-3}$ & $\mathrm{~kg} / \mathrm{kg}$ \\
\hline$k_{1}$ & 0.29268 & $\mathrm{KW} / \mathrm{K}$ & $T_{a d d}$ & $100.03+273.15$ & $\mathrm{~K}$ \\
\hline$k_{2}$ & 1.6207 & $\mathrm{KW}$ & $k_{3}$ & 0.073198 & $\mathrm{KW}$ \\
\hline$k_{4}$ & 31.111 & $\mathrm{KW}$ & $k_{5}$ & 0.26981 & $\mathrm{KW} / \mathrm{K}$ \\
\hline$k_{6}$ & -0.023367 & $\mathrm{KW} / \mathrm{K}$ & $D_{\text {diff }}$ & 0.091922 & \\
\hline
\end{tabular}

$$
\begin{aligned}
& y\left(t_{k}\right)=h\left(\bar{x}\left(t_{k}\right)\right)+v\left(t_{k}\right) \\
& z\left(t_{k}\right)=g\left(\bar{x}\left(t_{k}\right)\right)
\end{aligned}
$$

where $T_{s}=10 \mathrm{~s}$. The state and measurement noise covariances are $\bar{w}\left(t_{k}\right)=N_{i i d}\left(0, \bar{R}_{w}\right)$ and $v\left(t_{k}\right)=N_{i i d}\left(0, R_{v}\right)$. The two noise-terms are assumed to be uncorrelated. The system is simulated using a $4^{\text {th }}$ and $5^{\text {th }}$ order accurate Runge-Kutta method with variable step size.

\section{Model Predictive Control}

In this section we present the regulator and the state estimator in the linear MPC.

\section{A. Background and control objectives}

The controlled variables are the exhaust air temperature $T_{S D}$, the absolute air humidity, $Y$, and the product specific residual moisture content, $X . T_{S D}$ and $Y$ are closely related to the stickiness of the powder. The set-points for $T_{S D}$ and $Y$ are a result of empirical experience, and can only be exactly determined from product and spray dryer specific trials. Generally, there is a lower limit to $T_{S D}$ and an upper limit to $Y$. A simple RTO layer can be designed to provide such set-points depending on the type of product being dried. The set-point for $X$ is also product specific. The manipulated variables are the feed flow, $F_{f}$, the inlet main air temperature, $T_{\text {main }}$, and the inlet SFB air temperature, $T_{s f b}$. The inlet air flows, the feed concentration $X_{f}$, the feed temperature, $T_{f}$, the ambient air humidity, $Y_{a m b}$, and the indoor temperature, $T_{a m b}$, are all measured disturbances. The states and disturbances are measurable, also in industrial practice.

\section{B. Plant and Sensors}

The non-linear model of the dryer is numerically linearised, by applying small perturbations to the steady-state, and represented in state space form. To guarantee offset-free control of the outputs, $z$, we augment the process model with a number of integrating disturbances equal to the number of measured outputs $y$ [21]. Thus, the presence of constant unmeasured disturbances and plant model mismatch do not affect the tracking performance. The augmented model has the following form

$$
\begin{aligned}
x_{k+1} & =A x_{k}+B u_{k}+E d_{k}+\sigma_{x}+w_{k} \\
y_{k} & =C_{y} x_{k}+\sigma_{y}+v_{k} \\
z_{k} & =C_{z} x_{k}+\sigma_{z}
\end{aligned}
$$

with $x, u, d, y$ and $z$ being the same variables as in (8), but $x$ also including the disturbance states. $w$ and $v$ are distributed

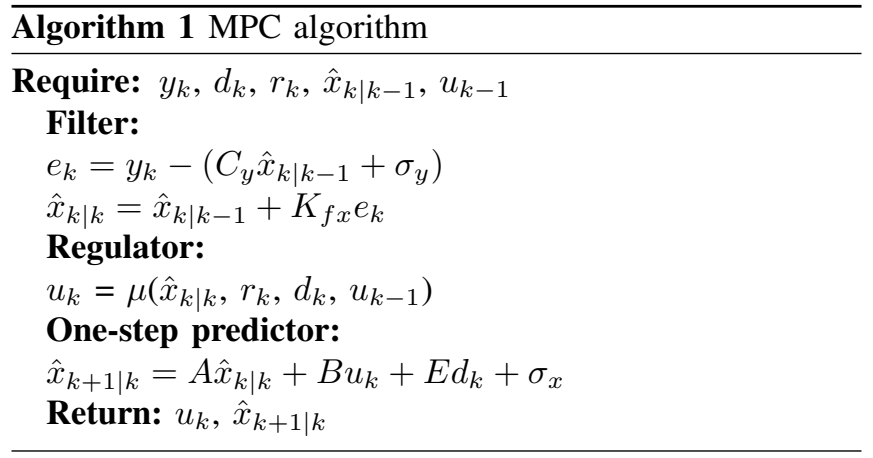

by $w_{k} \sim N_{i i d}\left(0, R_{w}\right)$ and $v_{k} \sim N_{i i d}\left(0, R_{v}\right)$. The initial state is, $x_{0} \sim N\left(\tilde{x}_{0}, P_{0}\right) . \sigma_{x}, \sigma_{y}$ and $\sigma_{z}$ contain the constants related to the linearisation of the model, i.e. $\sigma_{x}=x_{0}-A x_{0}-$ $B u_{0}-E d_{0}, \sigma_{y}=y_{0}-C_{y} x_{0}$ and $\sigma_{z}=z_{0}-C_{z} x_{0} .(A, B)$ is stabilizable and $\left(C_{y}, A\right)$ is detectable. Thus, we can control and estimate the states of the dryer.

\section{Regulator}

The output tracking problem with input and input rate constraints may be formulated as

$$
\begin{aligned}
& \min _{u_{k} \in \mathcal{N}_{u}} \phi \\
& \text { s.t. } \quad x_{k+j+1 \mid k}=A x_{k+j \mid k}+B u_{k+j \mid k} \\
& +E d_{k+j \mid k}+\sigma_{x}, \quad j \in \mathcal{N}_{u} \\
& z_{k+j \mid k}=C_{z} x_{k+j \mid k}+\sigma_{z}, \quad j \in \mathcal{N}_{z} \\
& u_{\text {min }} \leq u_{k+j \mid k} \leq u_{\max }, \quad j \in \mathcal{N}_{u} \\
& \Delta u_{\min } \leq \Delta u_{k+j \mid k} \leq \Delta u_{\max }, \quad j \in \mathcal{N}_{u}
\end{aligned}
$$

where

$$
\phi=\frac{1}{2} \sum_{j=1}^{N_{z}}\left\|z_{k+j \mid k}-r_{k+j \mid k}\right\|_{Q_{z}}^{2}+\frac{1}{2} \sum_{j=0}^{N_{u}}\left\|\Delta u_{k+j \mid k}\right\|_{S_{u}}^{2}
$$

in which $\Delta u_{k}=u_{k}-u_{k-1}$ and $\mathcal{N}_{z}=\left\{1,2 \ldots, N_{z}-1\right\}$, $\mathcal{N}_{u}=\left\{0,1 \ldots, N_{u}-1\right\}$. The control and prediction horizons are, $N_{z}=20 \mathrm{~min} / 10 \mathrm{~s}=120$ and $N_{u}=120$. These are selected sufficiently long such that any end effects have no influence on the solution in the beginning of the horizon. No forecasts are available for the references and disturbances, so we use the same-as-now forecasts, i.e. $r_{k+j \mid k}=r_{k}$ and $d_{k+j \mid k}=d_{k}$.

The problem in (14) can be converted to a constrained quadratic problem. Appendix A shows the details of the derivation of the regulator, the tuning parameters $Q_{z}$ and $S_{u}$ and the constraints. Algorithm 1 list the on-line computations in the linear MPC algorithm. The computation of $u_{k}=$ $\mu\left(\hat{x}_{k \mid k}, r_{k}, d_{k}, u_{k-1}\right)$ is performed by solving (14). The solution of the QP (14) is the computational expensive step in the MPC algorithm. The QP is solved using quadprog ()

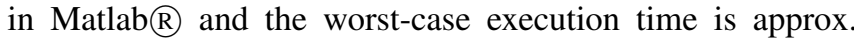
$12.5 \mathrm{msec}$.

\section{Closed-LOOP SIMULATIONS}

In this section we demonstrate the performance of the linear MPC and compare it to the performance of a conventional 


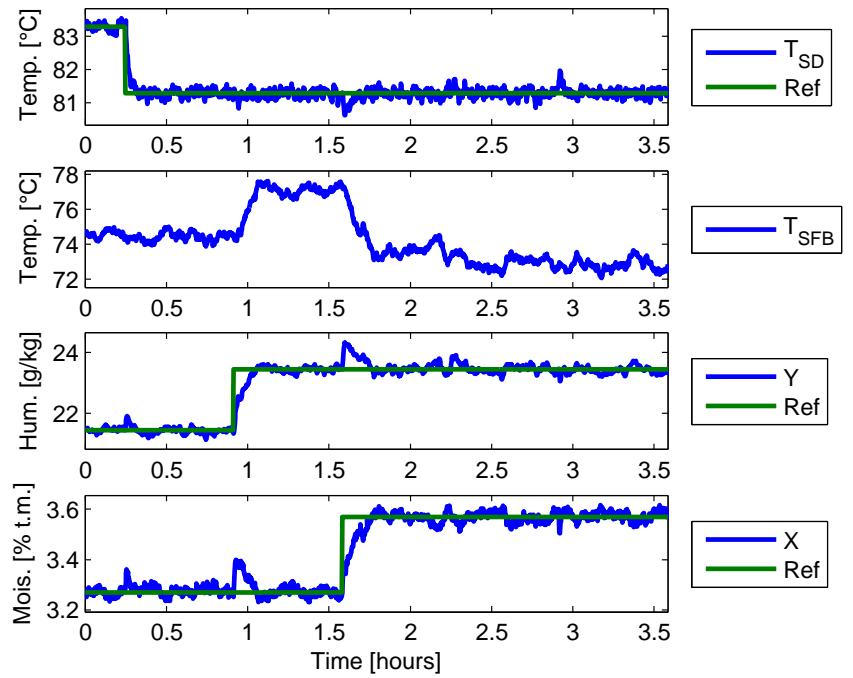

(a) Measured outputs of the system. The controlled variables are shown with the associated reference.

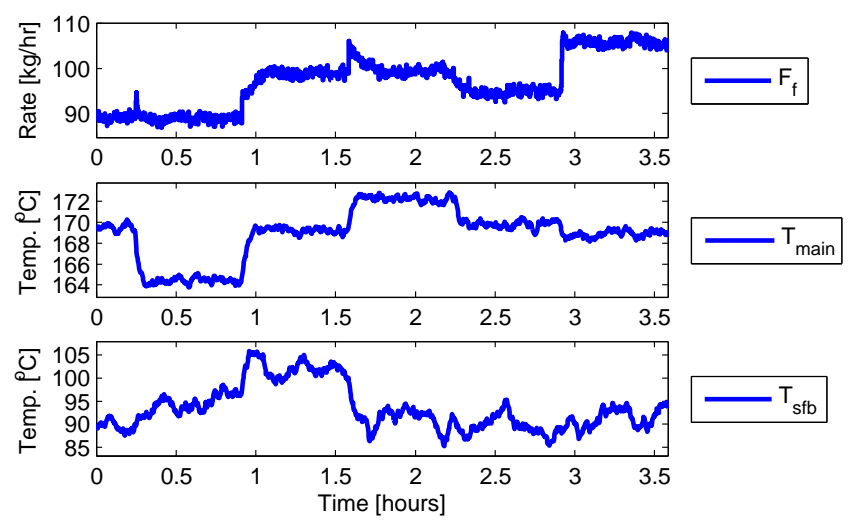

(b) Manipulated inputs to the system.

Fig. 3: Closed-loop simulation showing that the MPC tracks the set-points and rejects the unknown disturbances without steady-state error.

PID controller. The stochastic system model in (12) is used in the simulations.

\section{A. MPC Validation}

In the following the MPC will be validated against reference- and disturbance step changes. Fig. 3a shows the measured and controlled variables and Fig. $3 b$ shows the manipulated variables. The ambient air humidity changes from $3 \mathrm{~g} / \mathrm{kg}$ to $4 \mathrm{~g} / \mathrm{kg}$ at $t=2.25$ hours and the feed concentration decreases from $50.6 \%$ to $45.6 \%$ at $t=2.92$ hours. The disturbances are treated as unknown to the MPC in this simulation. At $t=0.25$ to $t=1.6$ three reference steps are introduced for each controlled variable. The exhaust air temperature and humidity, $T_{S D}$ and $Y$, change slightly faster than the residual moisture content, $X$. The MPC tracks the references and rejects disturbances without any offset. The system is controlled to a steady-state within reasonable time. The MPC handles the highly cross coupled system
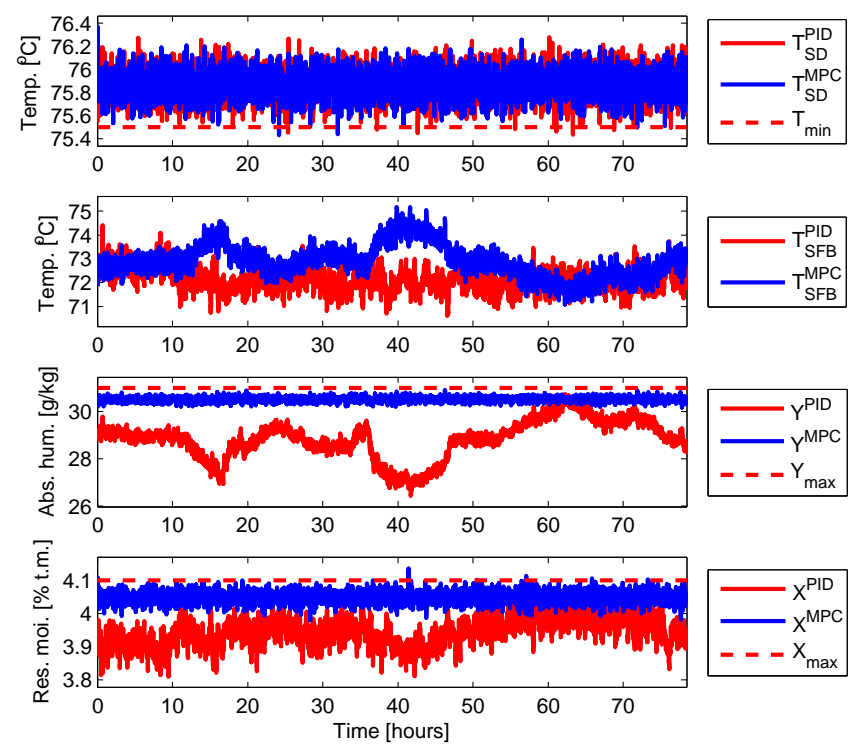

(a) Measured outputs of the system. The controlled variables are shown with the associated reference.

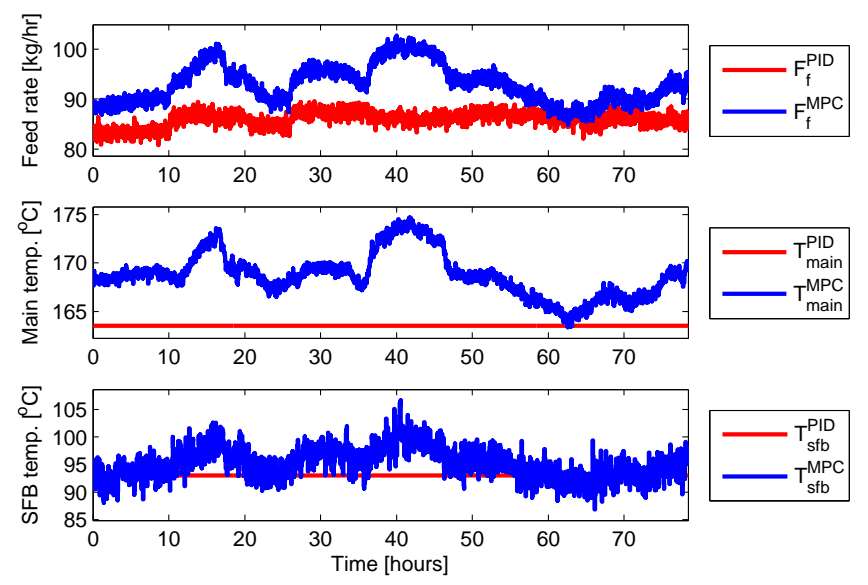

(b) Manipulated inputs to the system.
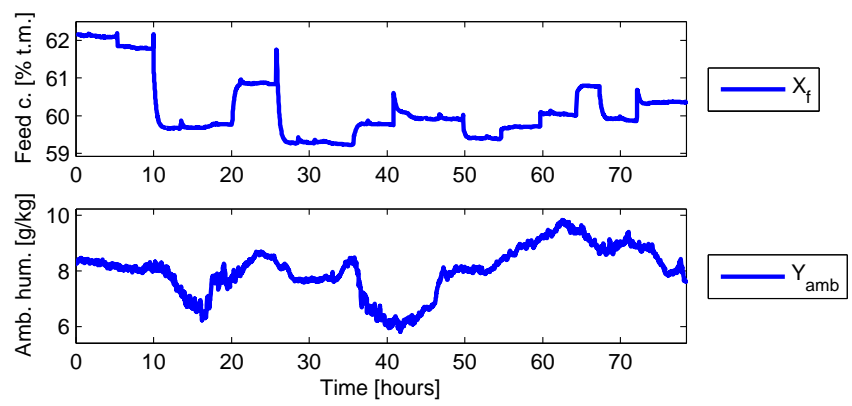

(c) Disturbance inputs to the system. The changes are used in feedforward to the controller i.e. $d_{k+j \mid k}=d_{k}$.

Fig. 4: Closed-loop simulation of an industrial disturbance scenario using an MPC and the conventional PID controller. The MPC tracks the set-points and rejects the disturbances. 

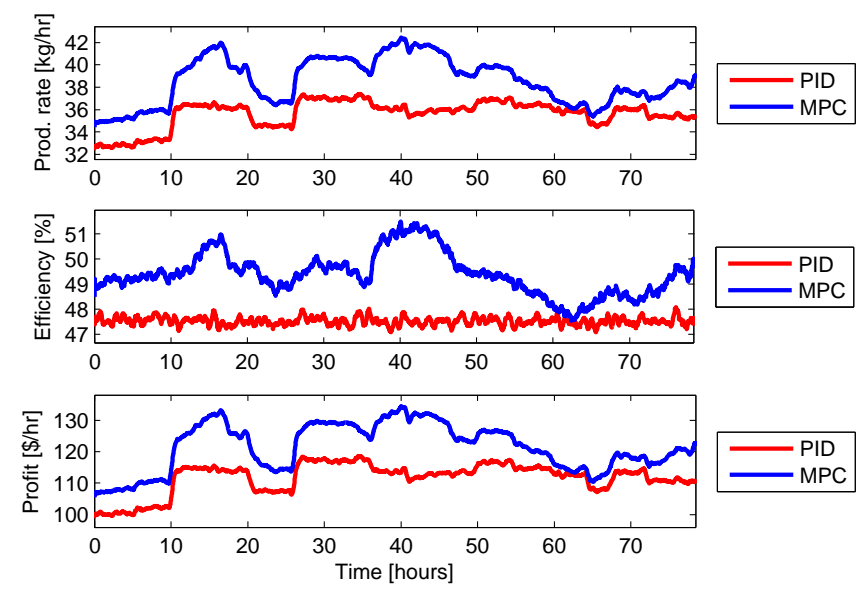

Fig. 5: The production rate, the energy efficiency and the profit of drying during the closed-loop simulation in Fig. 4.

dynamics well. The manipulated variables are kept within the input constraints and input rate constraints. The constraints are expected to become active occasionally as the load on the dryer is increased, i.e. as the feed flow, $F_{f}$, and the heat inputs, $T_{\text {main }}$ and $T_{s f b}$, are increased.

\section{B. Industrial scenario}

We also perform a closed-loop simulation with a realistic disturbance scenario from an industrial spray dryer. Thereby we can compare the achieved performance of the MPC to the conventional PID controller. Fig. 4 shows the spray dryer operation when controlled by the MPC and the conventional PID controller. Fig. 5 shows the KPIs. The disturbances are known to the MPC as these measurements are normally available in industry. The disturbances are recorded at a spray dryer producing whey protein concentrate in the period 8/13/2013 21:30 to 8/17/2013 04:00.

Fig. 4 shows the measured and controlled outputs, the manipulated variables and the disturbances. The simulation reveal that the proposed MPC is able to maintain a correct and constant residual moisture content, $X$, as well as maintaining the stickiness related variables, $T_{S D}$ and $Y$, at their setpoints. The MPC constantly adjusts the feed flow and inlet air temperatures to maintain the controlled variables at their set-points. An increase in the ambient air humidity increases the humidity in the dryer. Thus, the MPC must decrease the feed rate i.e. production. An increase in feed water concentration has the same effect and is also compensated by a decrease in the feed rate i.e. production.

The conventional PID controller maintains a constant exhaust air temperature, $T_{S D}$, by manipulating the feed rate, $F_{f}$. The two inlet air temperatures are fixed. With this control methodology, no correcting action is taken when the ambient air humidity increases. Changes related to the feed concentration are compensated for as the feed water affects the exhaust air temperature. Thus, on average the dryer need to dry the powder more than necessary to satisfy the specifications. The MPC only dries the powder to the specified level.
Fig. 5 shows the production rate, energy efficiency and profit of operation during the simulation. On average the MPC increases the production rate by $7.9 \%$, the energy efficiency by $4.1 \%$ and profit of production by $8.2 \%$. Thus, the dryer is used both more energy efficiently and increases the profit of operation significantly.

\section{CONCLUSIONS}

This paper presents a solution to the control problem of a two stage spray dryer. The controller is based on a set-point tracking MPC with output feedback. The states used in the regulator are estimated by a Kalman filter. The regulator and the estimator is designed based on the linearised non-linear system model. The simulations show that the air temperature and air humidity of the outlet air from the dryer can be controlled to a set-point as well as the residual moisture content in the powder. On average during the disturbance scenario the MPC increases the production rate by $7.9 \%$, the energy efficiency by $4.1 \%$ and the profit of production by $8.2 \%$. These numbers may be even greater in industry, as the conventional PID controller is often used more conservatively than presented in this paper. Thus, the dryer is used both more efficiently and increases the profit of operation significantly.

GEA Process Engineering A/S is currently implementing and testing the proposed control strategy at industrial scale spray dryers. The control methodology is generally applicable to other foods, chemicals and pharmaceuticals and not limited to two-stage dryers.

\section{REFERENCES}

[1] T. Donnellan, T. Hennessy, M. Fenelon, and D. O'Callaghan, “The potential for scale economies in milk powder processing: an irish case study," International Journal of Dairy Technology, vol. 67, no. 1, pp. 129-134, 2014.

[2] Vaisala. (2014, Feb.) $+20 \%$ increase in production capacity with zero increase in energy consumption. [Online]. Available: http://www.vaisala.com/Vaisala\%20Documents/Success\% 20Stories/CEN-TIA-G-Valio-Customer-Story-B211288EN-A.pdf

[3] D. O'Callaghan and P. Cunningham, "Modern process control techniques in the production of dried milk products - a review," Lait, vol. 85, pp. 335-342, 2005.

[4] L. Tan, F. Taip, and N. Aziz, "Simulation and Control of Spray Drying Using Nozzle Atomizer Spray Dryer." International Journal of Engineering \& Technology, no. 10, pp. 1-7, 2009.

[5] J. Pérez-Correa and F. Fariass, "Modelling and control of a spray dryer: a simulation study," Food Control, vol. 6, no. 4, pp. 219-227, 1995.

[6] L. N. Petersen, N. K. Poulsen, H. H. Niemann, C. Utzen, and J. B. Jørgensen, "A Grey-Box Model for Spray Drying Plants," 10th IFAC International Symposium on Dynamics and Control of Process Systems (DYCOPS 2013), pp. 559-564, 2013.

[7] D. O. Callaghan and P. Cunningham, "Modern process control techniques in the production of dried milk products a review," Lait, vol. 85, pp. 335-342, 2005.

[8] M. L. Darby, M. Harmse, and M. Nikolaou, "MPC: Current practice and challenges," IFAC Proceedings Volumes, vol. 7, no. 1, pp. 86-98, 2009.

[9] M. Bauer and I. K. Craig, "Economic assessment of advanced process control - a survey and framework," Journal of Process Control, vol. 18, no. 1 , pp. 2-18, 2008.

[10] M. R. Naysmith and P. L. Douglas, "Review of real time optimization in the chemical process industries," Developments in Chemical Engineering and Mineral Processing, pp. 67-87, 1995. 
[11] L. N. Petersen, N. K. Poulsen, H. H. Niemann, C. Utzen, and J. B. Jørgensen, "Economic optimization of spray dryer operation using nonlinear model predictive control," Proceedings of the IEEE Conference on Decision and Control, 2014.

[12] G. Prasath and J. B. Jørgensen, "Soft constraints for robust mpc of uncertain systems," International Symposium on Advanced Control of Chemical Processes, vol. 7, no. 1, pp. 225-230, 2009.

[13] J. B. Jørgensen, J. B. Rawlings, and J. K. Huusom, "Finite horizon mpc for systems in innovation form," Proceedings of the IEEE Conference on Decision and Control, pp. 1896-1903, 2011.

[14] J. K. Huusom, N. K. Poulsen, S. B. Jørgensen, and J. B. Jørgensen, "Tuning of methods for offset free mpc based on arx model representations," Proceedings of the American Control Conference, pp. 22552360, 2010.

[15] C. Hennigs, T. K. Kockel, and T. A. G. Langrish, "New measurements of the sticky behavior of skim milk powder," Drying Technology, vol. 19, no. 3-4, pp. 471-484, 2001.

[16] J. Smith, H. Van Ness, and M. Abbott, Introduction to chemical engineering thermodynamics, ser. McGraw-Hill chemical engineering series. McGraw-Hill, 2005.

[17] W. K. Lewis, "The rate of drying of solid materials," Journal of Industrial and Engineering Chemistry, vol. 13, pp. 427-432, 1921.

[18] F. F. G. Areed, M. S. El-Kasassy, and K. A. Mahmoud, "Design of neuro-fuzzy controller for a rotary dryer," International Journal of Computer Applications, vol. 37, no. 5, p. 34, 2012.

[19] C. E. C. Pérez, R. D. P. Andrade, and L. M. Roberto, "Models of sorption isotherms for food: Uses and limitations," Vitae, vol. 18, no. 3, pp. 325-334, 2011.

[20] C. G. J. Baker and K. A. McKenzie, "Energy consumption of industrial spray dryers," Drying Technology, vol. 23, no. 1-2, pp. 365-386, 2005.

[21] G. Pannocchia and J. B. Rawlings, "Disturbance models for offset-free model-predictive control," AIChE Journal, vol. 49, no. 2, pp. 426-437, 2003.

\section{APPENDIX}

In this section we briefly describe the details of the regulator design.

\section{A. Regulator}

The tracking problem in Sec. III-C is solved by formulating the corresponding convex quadratic problem. The tracking problem is similar to [12]-[14].

Define the vectors $Z, R, U$ and $D$ as

$$
\begin{gathered}
Z=\left[\begin{array}{c}
z_{k+1 \mid k} \\
z_{k+2 \mid k} \\
\vdots \\
z_{k+N_{z} \mid k}
\end{array}\right] \quad R=\left[\begin{array}{c}
r_{k+1} \\
r_{k+2} \\
\vdots \\
r_{k+N_{z}}
\end{array}\right] \quad U=\left[\begin{array}{c}
u_{k \mid k} \\
u_{k+1 \mid k} \\
\vdots \\
u_{k+N_{u} \mid k}
\end{array}\right] \\
D=\left[\begin{array}{llll}
d_{k \mid k} & d_{k+1 \mid k} & \ldots & d_{k+N_{z} \mid k}
\end{array}\right]^{T}
\end{gathered}
$$

Then the predictions are

$$
Z=\Phi_{x} x_{k \mid k-1}+\Gamma_{u} U_{k}+\Gamma_{d} D_{k}+\Omega \Sigma_{x}+\Sigma_{z}
$$

Using the predictions in vector form we can write the objective function as

$$
\begin{aligned}
\phi & =\frac{1}{2} \sum_{j=1}^{N_{z}}\left\|z_{k+j \mid k}-r_{k+j \mid k}\right\|_{Q_{z}}^{2}+\frac{1}{2} \sum_{j=0}^{N_{u}}\left\|\Delta u_{k+j \mid k}\right\|_{S_{u}}^{2} \\
& =\frac{1}{2}\left\|Z_{k}-R_{k}\right\|_{\mathcal{Q}_{z}}^{2}+\frac{1}{2}\left\|\Lambda U-I_{0} u_{k-1}\right\|_{S_{u}}^{2} \\
& =\frac{1}{2} U_{k}^{\prime} H U_{k}+g^{\prime} U_{k}+\rho
\end{aligned}
$$

with

$$
\begin{aligned}
H & =\Gamma_{u}^{T} \mathcal{Q}_{z} \Gamma_{u}+\Lambda^{T} \mathcal{S}_{u} \Lambda \\
g & =-\Gamma_{u}^{T} \mathcal{Q}_{z}\left(R_{k}-b\right)-\Lambda^{T} \mathcal{S}_{u} I_{0} u_{k-1} \\
\rho & =\frac{1}{2}\left\|-b-R_{k}\right\|_{\mathcal{Q}_{z}}^{2}+\frac{1}{2}\left\|I_{0} u_{k-1}\right\|_{S_{u}}^{2}
\end{aligned}
$$

where

$$
b=-\Phi_{x} x_{k \mid k}-\Gamma_{d} D_{k}-\Omega \Sigma_{x}-\Sigma_{z}
$$

The constraints are assumed constant over the prediction horizon and $u_{\min } \leq u_{k+j \mid k} \leq u_{\max }$ and $\Delta u_{\min } \leq$ $\Delta u_{k+j \mid k} \leq \Delta u_{\max }$ may be denoted

$$
\begin{aligned}
& U_{\text {min }} \leq U_{k} \leq U_{\text {max }} \\
& b_{l} \leq \Psi U_{k} \leq b_{u}
\end{aligned}
$$

where

$$
b_{l}=\Delta U_{\min }+I_{0} u_{k-1}, \quad b_{u}=\Delta U_{\max }+I_{0} u_{k-1}
$$

We solve the tracking problem by solution to the following convex quadratic problem

$$
\begin{array}{ll}
\min _{U_{k}} & \frac{1}{2} U_{k}^{T} H U_{k}+g^{T} U_{k}+\rho \\
\text { s.t. } & U_{\min } \leq U_{k} \leq U_{\max } \\
& b_{l} \leq \Psi U_{k} \leq b_{u}
\end{array}
$$

The MPC only apply the first $u_{0}^{*}$ of $U_{k}^{*}$ to the process. The open-loop optimization is repeated at the next sample where it also utilize the new state estimate $\hat{x}_{k}$.

The regulator is tuned to penalize deviation in the exhaust air temperature and humidity by 10 and the residual moisture content the most by 500 . Movements of the feed pump and inlet air temperatures are penalized, so that the feed pump is allowed to change very fast.

$$
Q_{z}=\operatorname{diag}\left(\left[\begin{array}{lll}
10 & 10 & 500
\end{array}\right]\right), \quad S_{u}=\operatorname{diag}\left(\left[\begin{array}{lll}
0.5 & 50 & 50
\end{array}\right]\right)
$$

The maximum capacity of the feed pump limits the feed flow. The inlet temperatures, $T_{\text {main }}$ and $T_{\text {sfb }}$ must be higher than the ambient temperature, $T_{\mathrm{amb}}$. Furthermore, the risk of powder explosions and the risk of scorched particles puts upper limits on the allowable inlet temperatures. Thus, we have

$$
\begin{aligned}
0 \mathrm{~kg} / \mathrm{hr} & \leq F_{f} \leq 200 \mathrm{~kg} / \mathrm{hr} \\
T_{\mathrm{amb}} & \leq T_{\text {main }} \leq 220^{\circ} \mathrm{C} \\
T_{\mathrm{amb}} & \leq T_{\mathrm{sfb}} \leq 120^{\circ} \mathrm{C}
\end{aligned}
$$

We do not impose input rate constraints. 